Alimentary Tract

\title{
Clinical efficacy, drug sustainability and serum drug levels in Crohn's disease patients treated with ustekinumab - A prospective, multicenter cohort from Hungary
}

\author{
Lorant Gonczi $^{\mathrm{a}}$, Kata Szanto ${ }^{\mathrm{b}}$, Klaudia Farkas ${ }^{\mathrm{b}}$, Tamas Molnar ${ }^{\mathrm{b}}$, Tamas Szamosi ${ }^{\mathrm{c}}$, \\ Eszter Schafer ${ }^{c}$, Petra A. Golovics ${ }^{c}$, Laszlo Barkai ${ }^{a}$, Livia Lontai ${ }^{a}$, Barbara Lovasz ${ }^{a}$, \\ Mark Juhasz $^{\mathrm{d}}$, Arpad Patai ${ }^{\mathrm{e}}$, Krisztina Sarang ${ }^{\mathrm{e}}$, Aron Vincze ${ }^{\mathrm{f}}$, Patricia Sarlos ${ }^{\mathrm{f}}$, \\ Alexandra Farkas ${ }^{\mathrm{g}}$, Zsolt Dubravcsik ${ }^{\mathrm{g}}$, Tamas G. Toth ${ }^{\mathrm{h}}$, Pal Miheller ${ }^{\mathrm{i}}$, Akos Ilias ${ }^{\mathrm{a}}$, \\ Peter L. Lakatos ${ }^{a, j, *}$
}

\footnotetext{
a Department of Medicine and Oncology, Semmelweis University, Budapest, Hungary

${ }^{\mathrm{b}}$ Department of Medicine, University of Szeged, Szeged, Hungary

${ }^{c}$ Department of Gastroenterology, Military Hospital-State Health Center, Budapest, Hungary

${ }^{\mathrm{d}}$ Department of Medicine, St. Margit Hospital, Budapest, Hungary

e Department of Medicine and Gastroenterology, Markusovszky Hospital, Szombathely, Hungary

${ }^{\mathrm{f}}$ First Department of Medicine, Medical School, University of Pecs, Pecs, Hungary

${ }^{g}$ Department of Gastroenterology, Bács-Kiskun County Hospital, Kecskemet, Hungary

${ }^{\mathrm{h}}$ Department of Gastroenterology, St. Janos Hospital, Budapest, Hungary

i 1st Department of Surgery and Interventional Gastroenterology, Semmelweis University, Budapest, Hungary

${ }^{\mathrm{j}}$ McGill University Health Center, Montreal General Hospital, Canada
}

\section{A R T I C L E I N F O}

\section{Article history:}

Received 23 April 2021

Accepted 13 July 2021

Available online $\mathrm{xxx}$

\section{Key words:}

Anti-TNF failure

Clinical efficacy

Crohn's disease

Drug sustainability

Ustekinumab

\begin{abstract}
A B S T R A C T
Introduction: Although efficacy of ustekinumab (UST) has been demonstrated through randomized trials, data from real-life prospective cohorts are still limited. Our aim was to evaluate clinical efficacy, drug sustainability, dose intensification and results from therapeutic drug monitoring in UST treated patients with Crohn's disease (CD) using a prospective, nationwide, multicenter cohort.

Methods: Patients from 10 Inflammatory Bowel Disease centers were enrolled between 2019 January and 2020 May. Patient demographics, disease phenotype, treatment history, clinical disease activity (Crohn's Disease Activity Index(CDAI), Harvey Bradshaw Index(HBI)), biomarkers, and serum drug levels were obtained. Evaluations were performed at week8 (post-induction), w16-20, w32-36, and w52-56 follow-up visits.

Results: A total of 142 patients were included [57.4\% female; complex disease behavior (B2/B3):48\%, previous anti-TNF exposition:97\%]. Clinical response and remission rates after induction(w8) were $78.1 \%$ and $57.7 \%$ using CDAI, and $82.5 \%$ and $51.8 \%$ based on HBI scores. The one-year clinical remission rate was 58\%/57.3\%(CDAI/HBI). Composite clinical and biomarker remission (CDAI $<150$ and C-reactive protein $<10 \mathrm{mg} / \mathrm{L}$ ) rates were $35.4 \% ; 33.3 \% ; 38.6 \%$ and $36.6 \%$ at $\mathrm{w} 8 / \mathrm{w} 16-20 / \mathrm{w} 32-36$ and $\mathrm{w} 52-56$. Drug sustainability was $81.9 \%$ (standard deviation(SD): 3.4 ) at 1 year(1y). Probability of dose intensification was high and introduced early, 42.2\%(SD:4.2) at $\sim \mathrm{w} 32$ and 51.9\%(SD:4.4\%) at $1 \mathrm{y}$.

Conclusion: Ustekinumab showed favorable drug sustainability and clinical efficacy in a patient population with severe disease phenotype and previous anti-tumor necrosis factor (anti-TNF) failure, however frequent dose intensification was required.
\end{abstract}

(c) 2021 Editrice Gastroenterologica Italiana S.r.l. Published by Elsevier Ltd. All rights reserved.
* Correspondence to: McGill University Health Centre, Montreal General Hospital, 1650 Ave. Cedar, D16.173.1, Montreal, QC H3G 1A4, Canada. Lakatos).

\section{Introduction}

Treatment options for inflammatory bowel diseases (IBD) have improved substantially in recent years with the introduction of 
anti-TNF agents and selective anti-integrin therapy, however, a large portion of patients with refractory disease (primary and secondary non-responders) still require novel therapeutic options. Ustekinumab is a fully human, immunoglobulin G1 monoclonal antibody that targets the standard p40 subunit of the IL-12 and IL-23 cytokines. By binding p40, the activity of the IL-23 and IL-23 receptors, which are found on $\mathrm{T}$ cells, antigen-presenting cells and natural killer cells, is blocked, thus effecting a negative feedback on chronic immunological responses [1].

Ustekinumab demonstrated good efficacy in inducing clinical and endoscopic remission in moderate-to-severe Crohn's disease (CD) patients in the UNITI 1 and UNITI 2 randomized controlled trials (RCTs), meanwhile, long-term efficacy and safety data of 96 weeks follow-up have been reported in IM-UNITI [2,3]. Furthermore, good efficacy in the TNF $\alpha$ failure population was also shown in the CERTIFI trial [4].

Although efficacy and safety of ustekinumab have been well demonstrated through RCTs, patients enrolled in clinical trials are not entirely representative of those treated everyday clinical practice, thus data from real-life prospective cohorts are still of great interest. Real-world experience allows bridging of some data gaps that are lacking in RCTs, adding substantial information to clinical practice on safety, efficacy, optimal treatment interval and dosing based on unselected patient populations.

Ustekinumab had been approved by both the US Food and Drug Administration and the European Medicines Agency for the treatment of CD in 2016, and it was adopted for general reimbursement by the National Health Insurance Fund of Hungary (NEAK) in October 2018.

The aim of the present study was to evaluate the clinical efficacy, drug sustainability, frequency of dose intensification, and results from therapeutic drug monitoring in ustekinumab treated Crohn's disease patients based on a multicenter prospective cohort from Hungary.

\section{Materials and methods}

\subsection{Study design and patients}

This is a prospective multicenter observational study conducted at 10 Hungarian referral IBD centers (5 academic centers and 5 county hospitals). Eligible patients with an age of 18 years or older and receiving ustekinumab therapy were consecutively enrolled between January 2019 and May 2020.

For induction treatment, patients were given a single dose of ustekinumab intravenous injection using a weight-based dosage regimen: $260 \mathrm{mg}<55 \mathrm{~kg}, 390 \mathrm{mg}$ between $55 \mathrm{~kg}$ and $85 \mathrm{~kg}$, and $520 \mathrm{mg}>85 \mathrm{~kg}$. This was followed by subcutaneous (s.c.) injections (90 mg) starting at week 8 (w8), and followed by s.c. injections every 12 weeks, constituting the maintenance treatment. In case of dose-intensification, s.c. injections were due every 8 or every 4 weeks.

A standardized monitoring strategy was applied in all participating centers, as requested by the Hungarian National Health Fund. Data of patient demographics, disease phenotype, treatment history (surgical history, previous and present concomitant medications) were collected from the electronic medical records and upon patient inclusion. Disease location and behavior were assessed according to the Montreal classification [5].

Primary outcomes of the present study were evaluation of clinical disease activity and drug sustainability after a one-year followup period. Secondary outcomes included corticosteroid-free remission rates, composite clinical and biomarker remission rates and dose intensification rates. Data on serum drug levels in available samples were also collected.
Evaluations of clinical disease activity and collection of data on biomarkers and therapeutic drug monitoring (if available) were performed at baseline, w8 (post-induction), w16-20, w32-36, and w52-56 (1-y) follow-up visits. Clinical disease activity was evaluated using the Crohn's Disease Activity Index (CDAI) and Harvey Bradshaw Index (HBI) [6,7]. Infusion and injection related adverse events were registered at baseline and at every follow-up visit.

Baseline clinical disease activity was defined moderate-tosevere $(\mathrm{CDAI}>220$ or $\mathrm{HBI}>7)$, mild $(150 \leq \mathrm{CDAI} \leq 220$ or $5 \leq \mathrm{HBI} \leq 7)$, or clinical remission (CDAI $<150$ or $\mathrm{HBI}<5$ ) based on these indices. Clinical response was defined as a decrease of CDAI by $\geq 70$ points or decrease of $\mathrm{HBI} \geq 3$ points, respectively. Clinical remission was defined as a CDAI of less than 150 points, or an HBI score of less than 5 points. We chose to present the two most commonly used clinical activity scores in CD simultaneously, as a sensitivity analysis of our results.

In fistulizing disease, remission was defined as no fistula drainage. Biomarker evaluation consisted of C-reactive protein (CRP) measurement. Cut-off level for CRP was set at $<10 \mathrm{mg} / \mathrm{L}$. The definition of corticosteroid-free remission was clinical remission based on CDAI/HBI without any systemic corticosteroid medication at the time of the assessment. We calculated 'composite clinical and biomarker remission' rates, defined by clinical remission based on CDAI/HBI scores and CRP levels below cut-off.

Serum drug trough level (TL) and anti-drug antibody (ADA) were measured using enzyme-linked immunosorbent assay methods (ELISA) by Lisa-Tracker ustekinumab Duo kit. (Theradiag, France). All sample measurements were performed at the Department of Laboratory Medicine, Semmelweis University, Budapest.

\subsection{Statistical analysis}

For the characterization of demographic data, remission and response rates, adverse events descriptive statistics were applied. Medians and interquartile ranges (IQR) were calculated for continuous variables. Kaplan-Meier survival curves were used to evaluate drug sustainability and dose escalation. Log-rank test was used to compare dose-escalation probabilities in patient subgroups. Statistical analysis was performed using SPSS software v. 20.0 (Chicago, IL); $P<0.05$ was considered statistically significant.

\subsection{Ethical considerations}

Ethical approval of the study was acquired from the Hungarian Medical Research Council's Committee of Scientic and Research Ethics [ETT-TUKEB 20877-1/2019/EKU]. Consent forms of all patients were obtained in accordance with the Helsinki Declaration.

\section{Results}

\subsection{Description and initial clinical characteristics of the cohort}

A total of $142 \mathrm{CD}$ subjects were consecutively included with a median follow-up time of 60 weeks (IQR: $47.5-79.5$ ), $56.3 \%$ of them were female, mean age was $38.4 \pm 13.0$ years. Complex disease phenotype (B2-B3) was present in 48.2\%, whereas ileocolonic disease location (L3) in $55.7 \%$, and perianal manifestation in $46.8 \%$ of the patients. Prior resective and perianal surgical history was $45.4 \%$ and $46 \%$, respectively. Previous anti-TNF exposition was $97.2 \%$, while previous vedolizumab failure was $25.5 \%$. Detailed patient characteristics are shown in Table 1.

Median clinical disease activity score at baseline was 270 (IQR: 189-323) based on CDAI, and 10 (6-15) using HBI score. $66.2 \%$ / $66.9 \%$ of the patients had moderate-to-severe clinical disease activity at inclusion ( $\mathrm{CDAI}>220 / \mathrm{HBI}>7)$. $19.7 \%$ / $16.9 \%$ of the study population had mild clinical disease activity at inclusion 
Table 1

Baseline characteristics of the study individuals.

\begin{tabular}{|c|c|}
\hline & $\mathrm{CD}(n=142)$ \\
\hline Gender (male/female; n) & $62(43.7 \%) / 80(56.3 \%)$ \\
\hline Age at disease onset (mean (SD); y) & $25.6(11.0)$ \\
\hline Age at inclusion (mean (SD); y) & $38.4(13.0)$ \\
\hline Disease duration (median (IQR); y) & $12.5(8.0-18.0)$ \\
\hline \multicolumn{2}{|l|}{ Age at diagnosis (\%) } \\
\hline A1 & 19.0 \\
\hline $\mathrm{A} 2$ & 69.7 \\
\hline A3 & 11.3 \\
\hline \multicolumn{2}{|l|}{ Smoking (\%) } \\
\hline NO & 74.2 \\
\hline YES & 18.8 \\
\hline past smoker & 7.0 \\
\hline \multicolumn{2}{|l|}{ Location (\%) } \\
\hline L1 & 17.1 \\
\hline $\mathrm{L} 2$ & 22.8 \\
\hline L3 & 55.7 \\
\hline L4 & 6.5 \\
\hline \multicolumn{2}{|l|}{ Behavior (\%) } \\
\hline B1 & 51.8 \\
\hline B2 & 25.5 \\
\hline B3 & 22.7 \\
\hline Perianal manifestation (\%) & 46.8 \\
\hline Previous surgical resection (\%) & 45.4 \\
\hline Previous perianal surgery (\%) & 46.0 \\
\hline Previous immunosuppressive therapy (\%) & 80.9 \\
\hline \multicolumn{2}{|l|}{ Previous anti-TNF therapy (\%) } \\
\hline NO previous anti-TNF & 2.8 \\
\hline IFX & 14.2 \\
\hline ADA & 19.9 \\
\hline $\mathrm{IFX}+\mathrm{ADA}$ & 63.1 \\
\hline Previous vedolizumab therapy (\%) & 25.5 \\
\hline $\begin{array}{l}\text { Concomitant immunosuppressive therapy at } \\
\text { baseline (\%) }\end{array}$ & 20.2 \\
\hline Concomitant corticosteroid therapy at baseline (\%) & 34.0 \\
\hline Median follow-up time (IQR; weeks) & $60(47.5-79.5)$ \\
\hline
\end{tabular}

(CD: Crohn's disease, SD: standard deviation, IQR: interquartile range, anti-TNF: anti-tumor necrosis factor, IFX: infliximab, ADA: adalimumab)

$(150<\mathrm{CDAI}<220 / 5 \leq \mathrm{HBI} \leq 7)$, and $14.1 \%$ / $16.1 \%$ were in clinical remission $(\mathrm{CDAI}<150 / \mathrm{HBI}<5)$ at inclusion. Of note, parallel systemic corticosteroid medication was present in as high as $34.0 \%$ of the patients at baseline.

\subsection{Clinical response and remission rates}

Eight weeks after the induction therapy, $78.1 \%$ of the total study population showed clinical response as per CDAI, and $57.7 \%$ were in clinical remission. As per HBI these numbers were $82.5 \%$ and $51.8 \%$, respectively. When separately analyzing patients with moderate-to-severe disease activity at baseline, clinical response rates were $78.9 \% / 78.9 \%$ using CDAI or HBI scores, however only $50.0 \%$ / 35.5\% (CDAI/HBI) of these individuals were in clinical remission. During the maintenance phase, clinical remission rates at half year (week 32-36) were 54.9\% / 56.5\%, while one year clinical remission rates were 58\% / 57.3\% (CDAI/HBI). For detailed results see Fig. 1. Clinical remission rates in patients with moderate-to-severe disease activity at baseline were consequently lower throughout the study period, 50\% / 35.5\% at w8; 62.6\% / $50.6 \%$ at w16-20; $46.9 \%$ / $44.3 \%$ at w32-36; and $47.1 \%$ / $50 \%$ at one year (CDAI/HBI). In a subgroup-analysis, patients with mild or no clinical disease activity at baseline presented clinical remission rates of $72.3 \%$ / 84.2\% at w8; $70.7 \%$ / 83.8\% at w32-36; and $58.6 \%$ / $68.4 \%$ at one year (CDAI/HBI).

Notably, a significant proportion of patients received concomitant systemic corticosteroid therapy at baseline (34.0\%), and $26.3 \%$ at post-induction (w8). At week $32-3621.0 \%$, and at one year still $16.9 \%$ of the patients received systemic corticosteroids. (see Supplementary Table 1). Steroid-free clinical remission rates of the total cohort are detailed in Fig. 2. In patients with moderate-tosevere disease activity at baseline, remission rates were consequently lower: $33.3 \%$ | 24.3\% at w8; $53.4 \%$ | $43.6 \%$ at w16-20; 40.3\% / 39.1\% at w32-36; and 50.8\% / 47.5\% at one year (CDAI/HBI).

We also evaluated drug efficacy using a combined criteria of clinical score remission and normalized biomarker levels (CRP). Composite clinical and biomarker remission rates $(\mathrm{CDAI}<150$ and $\mathrm{CRP}<10 \mathrm{mg} / \mathrm{L}$ ) were $35.4 \%, 33.3 \%, 38.6 \%$, and $36.6 \%$ by week 8 , w1620, w32-36, and w52-56, respectively (Fig. 3). Composite clinical and biomarker remission rates in patients with moderate-to-severe disease activity at baseline were as follows: $30.7 \%, 29.1 \%, 31.9 \%$, and $40 \%$ by week 8 , w16-20, w32-36, and w52-56, respectively.

Analyzing the patients according to their prior biological therapy revealed a tendency to lower clinical efficacy in case of multiple previously failed biologicals. For detailed composite clinical and biomarker remission rates stratified by previous biological exposure see Supplementary Fig. 1.

\subsection{Dose intensification and drug sustainability}

Drug sustainability was high with $90.4 \%$ (SD: 2.5 ) of the patients remaining on treatment at week 32 , and $81.9 \%$ (SD: 3.4 ) at one year. (Fig. 4/A) Overall $n=23$ patients discontinued therapy during our follow-up (loss of response: $n=14$; pregnancy: $n=4$; side effect: $n=2$; lost to follow-up: $n=3$ ). Probability of dose intensification ( $\mathrm{Q} 8$ or $\mathrm{Q} 4$ regimens) was high and introduced early in the treatment, $42.2 \%$ (SD: 4.2 ) at week 32 and 51.9\% (SD: 4.4\%) at one year (Fig. 4/B). Q8 dosing regimens were introduced in 61 patients (43.0\%) during our follow-up, while 16 patients (11.3\%) received a subcutaneous dose every 4 weeks. Patients with complex disease phenotype (B2/B3) had higher probability for dose intensification (Log-rank: $4.17 ; p=0.042$ ). A similar trend could be observed in patients with perianal manifestation, who required dose intensification more frequently (Log-rank: $2.22 ; p=0.136$ ).

\subsection{Adverse events}

No serious adverse events were observed in our cohort. Two subjects discontinued therapy due to adverse events: one case of arthralgia and another patient with recurrent skin erythema at the injection site. Skin reaction at injection site was reported by two other patients, and one patient reported hair loss. All three patients continued treatment. No allergic reactions were detected at the time of intravenous infusions.

\subsection{Therapeutic drug monitoring}

Data from $n=58$ serum drug level and anti-drug antibody measurements were available. Mean serum trough levels of ustekinumab were 4,28 $\pm 3,35 / 1,35 \pm 1,42 / 0,82 \pm 0,65$ and $1,13 \pm 0,74 \mu \mathrm{g} / \mathrm{mL}$ measured at $\mathrm{w} 8 / \mathrm{w} 16-20 / \mathrm{w} 32-36$ and $\mathrm{w} 52-56$ follow-up visits. (Fig. 5) ADAs were exceeding $1 \mathrm{AU} / \mathrm{mL}$ in only 2 patients.

\section{Discussion}

The results of this current prospective multicenter study provide additional post-marketing data on the efficacy and safety of ustekinumab in unselected patients of a 'real-world' setting. We also show data on the medium-term drug sustainability and the probability of dose-intensification in a heavily burdened patient population with a high rate of complex disease phenotype and virtually $100 \%$ of previous anti-TNF treatment failure.

Ustekinumab showed good short- and medium-term clinical response and remission rates, corticosteroid-free and composite 

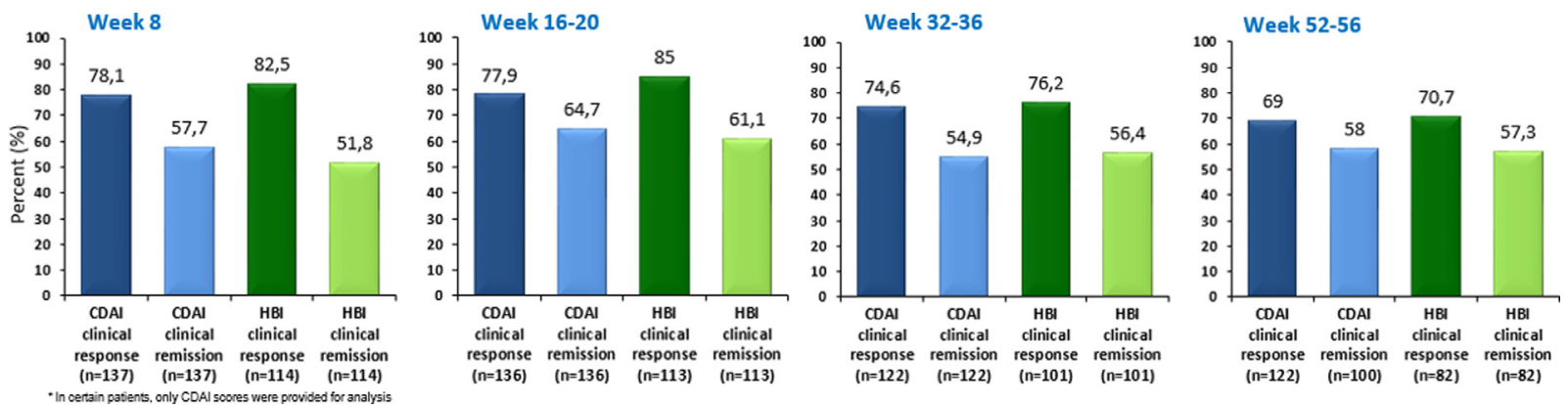

Fig. 1. Clinical response and remission rates based on CDAI and HBI scores.
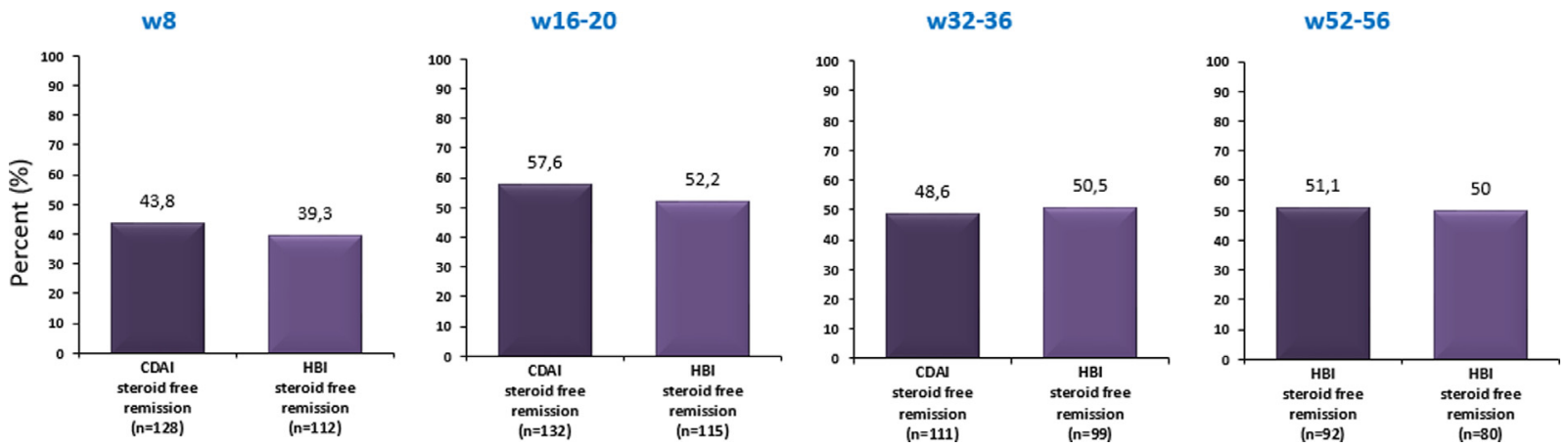

Fig. 2. Corticosteroid-free clinical remission rates.
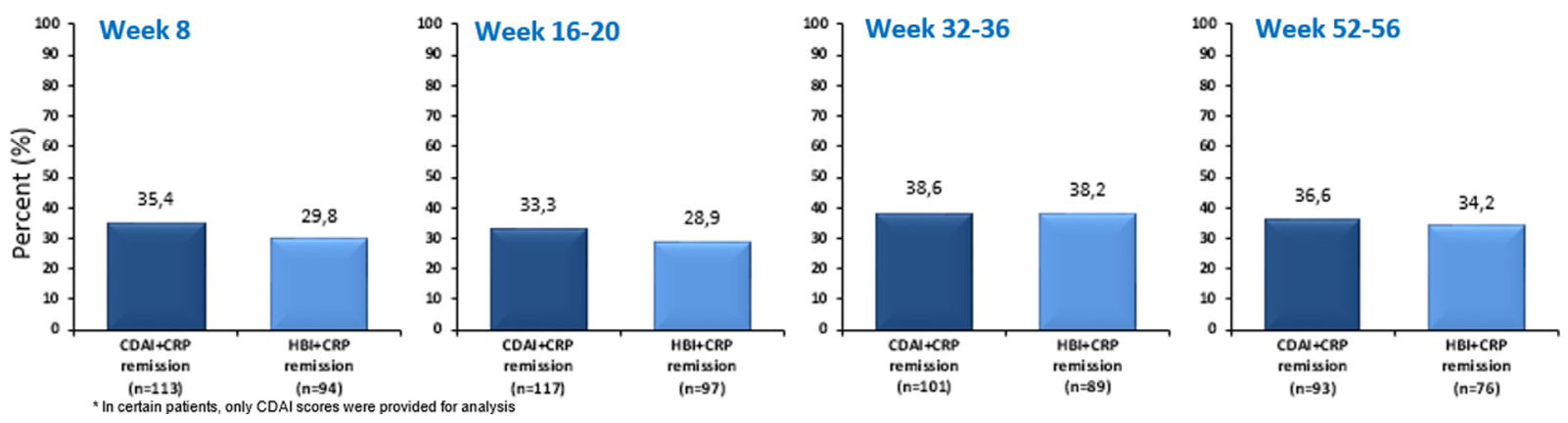

Fig. 3. Composite clinical and biomarker remission rates (CDAI / HBI score remission and CRP<10 mg/L).

A

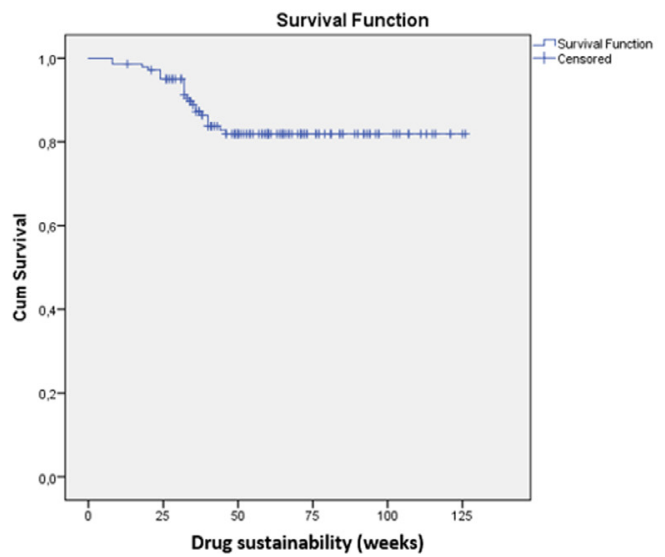

B

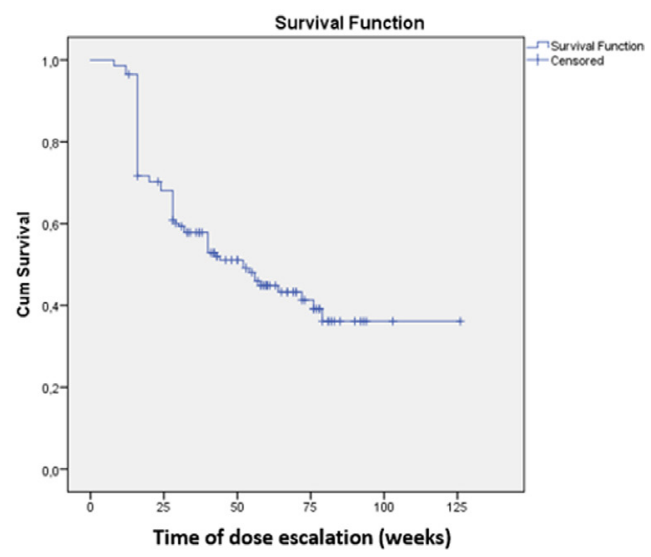

Fig. 4. Drug sustainability (A) and probability of dose intensification (B) in Kaplan-Meier analyzis. 


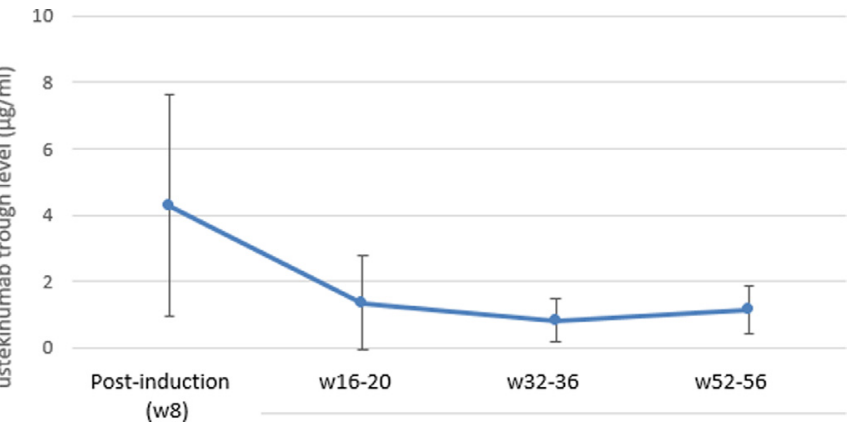

Fig. 5. Mean serum trough levels of ustekinumab.

(clinical + biomarker $/ \mathrm{CRP} /$ ) remission rates in this biologicexposed population. After 52 weeks, $58.0 \%, 51.1 \%$ and $36.6 \%$ of all patients were in clinical, corticosteroid-free clinical, and combined clinical and biomarker (CRP) remission, respectively. In comparison, in the IM-UNITI registration trial, $41.1 \%$ achieved clinical remission at week 44 using anti-TNF refractory patients only (from UNITI-1). Corticosteroid-free remission rates were $46.9 \%$ at week 44 of the total population (IM-UNITI) [2]. In the CERTIFI clinical trial of anti-TNF resistant Crohn's disease patients, glucocorticoid-free remission at week 22 was 30.6\% [4]. However, only patients with moderate-to severe baseline clinical activity and a clinical response at post-induction were included in these analyses as a consequence of clinical trial design, in contrast to our 'real-life' cohort that analyzed all patients.

When interpreting remission rates in clinical efficacy using 'real-life' cohorts, one must take into account the proportion of patients with mild or no clinical disease activity at baseline. In our cohort, baseline disease activity was mild or in remission up to $\sim 35 \%$ of the patients. This relatively high rate of patients with mild or no symptomatic disease activity can be explained by the use of concomitant corticosteroid to treat disease flare, before the initiation of biological therapy, or by biological treatment change due to adverse events, or simply by the known discrepancies between endoscopic / biomarker disease activity and clinical symptoms in some patients.

Our results are comparable to published data from other unselected, mainly retrospective studies, however, prospective 'real-life' cohorts are still limited, to date. A prospective multicenter study from the Netherlands included $221 \mathrm{CD}$ patients $(98.6 \%$ anti-TNF and $46.6 \%$ vedolizumab exposed) with a median followup of 52 weeks. At baseline, a proportion of the patients (30.8\%) were in clinical remission based on the clinical indices, but had biomarker or endoscopic activity, similarly to the present cohort. Corticosteroid-free clinical remission rates in this study were comparable to our results, and similar sub-analyses were carried out excluding patients without clinical activity at baseline. For patients with active disease at baseline, remission rates were $24.2 \%, 38.2 \%$ and $37.1 \%$ at weeks 12,24 and 52, respectively. When analyzing all patients regardless of clinical disease activity at baseline [ $n=221]$, the proportion of patients in corticosteroid-free clinical remission at weeks $0,12,24$ and 52 were $25.8 \%, 35.7 \%, 46.6 \%$ and $41.8 \%$, respectively [8]. Very recently, the two-year effectiveness and safety results of this prospective Dutch registry have been published [9]. Of all included patients [ $n=252$ ], corticosteroid-free clinical remission rates were $32.3 \%, 41.4 \%, 39 \%$ and $34.0 \%$, at week 12,24 , 52 and 104, respectively. In patients with combined clinical and biomarker disease activity at baseline $[n=122]$ the corticosteroidfree clinical remission rates were $23.8 \%, 35.2 \%, 40.0 \%$ and $32.8 \%$ at week 12, 24, 52 and 104, respectively. These figures correlate well with our findings, namely around $\sim 40 \%$ of the patients reach steroid-free remission at 1 year. The probability of remaining on ustekinumab treatment after 52 and 104 weeks in all patients was $64.3 \%$ and $54.8 \%$, and no new safety issues were observed.

A smaller Canadian prospective cohort $[n=62]$ of antiTNF refractory or intolerant patients showed very good clinical effectiveness (66.1 and 50.0\% clinical and corticosteroid-free clinical remission at week 26), however, a very high portion of patients were on a q4w dosing interval (77.4\%), and no antiintegrin exposed patients were included [10]. A recent prospective, multicenter Israeli study evaluated short-term clinical outcomes in ustekinumab-treated CD patients. Both anti-TNF and vedolizumab exposure was as high as $63 \%$ in this cohort. Clinical response was observed in $52 \%$, while clinical remission was achieved in $31.1 \%$ of the patients at week 24 [11]. More recently, results from a Sicilian $\mathrm{CD}$ cohort were published with a total of 131 patients. Steroid-free clinical remission rates at 24 and 52 weeks of follow-up were $40 \%$ (47/117) and 43\% (33/76) [12].

In addition, a large retrospective multicenter national cohort study from Belgium included 152 patients, all having been previously exposed to at least one anti-TNF agent, and $69.7 \%$ to even two anti-TNF and vedolizumab. After 1 year, clinical efficacy results were more modest, with $25.7 \%$ of patients experiencing clinical remission, and $24.3 \%$ steroid-free clinical remission, respectively [13]. Another retrospective chart review study, called FINUSTE2, captured data at 17 Finnish hospitals ( $n=155 \mathrm{CD}$ patients, $\sim 66 \%$ exposed to two or more biological) and reported a corticosteroidfree biomarker remission rate of $41.4 \%$ at 1 year [14]. Finally, the Spanish retrospective ENEIDA registry presents results on a total of 407 patients, of whom only $4 \%$ had not been previously treated with anti-TNF $\alpha$ agents. Of the patients with clinical disease activity at baseline $(n=295), 57 \%$ and $64 \%$ achieved clinical remission at weeks 26 and 52, respectively [15].

A systematic review and pooled analysis of retrospective studies were carried out by Engel et al. on the real-world efficacy of ustekinumab in anti-TNF failure patients. 578 patients of 6 eligible studies were pooled for analysis, excluding RCT cohorts and studies missing well-reported efficacy data on long-term maintenance. Pooled clinical response rate was $60 \%, 62 \%$, and $49 \%$ at 12,24 and 52 weeks, respectively, while pooled clinical remission rate was $39 \%$ at 24 weeks and $28 \%$ at 52 weeks (data of only two studies, not adequate for pooled statistical analysis). Steroid-free response rates, based on 4 cohorts with a total of 261 patients, yielded a pooled proportion of $51 \%$ at one year [16].

It is important to point out that relatively high rates of concomitant systemic corticosteroid therapy were observed in our study with $34 \%$ of the patients receiving steroids at baseline, $26 \%$ at the end of induction, and $17 \%$ after one year. However, similarly high steroid need was observed in other cohorts comprising patients with multiple previous biological treatment failures. In a Spanish multicenter cohort, $35.1 \%$ of the patients received systemic corticosteroids at the time of ustekinumab introduction [17]. In the Israeli prospective cohort, $34.9 \%$ of the patients were treated with systemic steroids at induction, which decreased to $15 \%$ at week 24 [11]. In another multicenter cohort from Belgium, concomitant steroids at induction were high with $44.7 \%$ at baseline [including $70.1 \%$ of systemic steroids (methylprednisolone) and $29.9 \%$ of oral controlled-release formulation (beclomethasone or budesonide)]. By one year of follow-up, a progressive steroid weaning was observed: $34.2 \%$ at week $8,17.1 \%$ at week 16 and $11.2 \%$ at week 52 [13].

An advantage of the present study was that composite clinical and biomarker remission rates were provided using CRP levels to more accurately identify patients in remission. The rate of composite remission was $\sim 35 \%$ post-induction, which was then maintained during the one-year follow up. Very few studies provide similar endpoints, one of them is the previously mentioned prospective multicenter study by Biemans et al., which applies 
even stricter criteria by using steroid-free remission and a cut-off value of $\leq 5 \mathrm{mg} / \mathrm{L}$ for CRP. The proportion of patients in combined corticosteroid-free clinical and biomarker remission at weeks 12 , 24 and 52 was $5.2 \%, 13.9 \%$ and $18.2 \%$, respectively [8]. We also stratified composite clinical and biomarker remission rates by previous biological exposure and showed trends to progressively weakening efficacy, as expected in a population with multiple biological failures.

We detected very high rates of drug sustainability in our cohort, with $82 \%$ of the patients remaining on treatment after the first year. Lower rates were reported in the prospective cohort by Biemans et al. The probability of remaining on ustekinumab treatment after 52 weeks was $62.9 \%$ in survival analysis [8]. A multicenter retrospective cohort from Spanish IBD centers included therapy resistant CD patients $(n=116)$, with 2 or more previous anti-TNF failures in $87 \%$ of the subjects. The cumulative probability for drug survival at 6 and 12 months was $86 \%$ (95\% CI: 83.1-88.9) and $74 \%$ (95\% CI: 70.3-77.7), more similar to the present study [17].

The rate of dose intensification was high and introduced early in the treatment, with $42.2 \%$ of the patients receiving Q8 or Q4 regimens at week 32 in our cohort. Other cohorts of patients with multiple previous treatment failures present even higher dose escalation rates. In the Dutch cohort discussed above, at week 12 already $65.0 \%$ continued their maintenance interval with injections every 8 weeks or less. At week $24,77.3 \%$, while at week $52,85.2 \%$ of the patients received the injection every 8 weeks or less [8]. Also very high rates of dose escalation are reported in studies from Spain and Canada [17,10]. In a meta-analysis of earlier / retrospective cohorts, dose escalation rates reached only a pooled rate of $15.6 \%$ [16]. Of note, comparing dose intensification rates is very difficult, as it is dependent on the disease severity of the cohorts in question and also sensitive to reimbursement regulations constantly changing by countries and time.

'Real-world' evidence data often suggest higher clinical efficacy and drug survival rates compared to RCTs. This discrepancy is most likely due to a less strict definition of clinical response/benefit in 'real-world' studies. For many patients in this population, ustekinumab was the 'last resort' therapeutic option. Of note, the availability of vedolizumab in our study was somewhat limited due to reimbursement regulations in Hungary, therefore previous exposure to vedolizumab was relatively low ( $25 \%)$. Almost $50 \%$ of the patients had a stricturing or penetrating disease behavior, and $45 \%$ had perianal manifestations (21.3\% having a seton at baseline). Under these circumstances, it is somewhat natural that ustekinumab treatment could have been extended in some patients even without clear evidence of benefit, and treatment discontinuation rates could have been higher if additional lines of therapy were available.

No serious adverse events, and only two instances of discontinuation due to adverse events were observed in our cohort, which correlates the favorable safety profile of ustekinumab previously reported 8,9,11]. Information on serum drug levels and anti-drug antibodies was only available in a limited number of patients in the present cohort. Recent data of meta-analysis from Phase 3 studies suggest that drug levels were proportional to Q12 and Q8 dosing regimen and reached a steady state by the second maintenance dose. Trough concentrations of ustekinumab of $0.8 \mathrm{~g} / \mathrm{mL}$ or greater were associated with maintenance of clinical remission. UST antibodies were found in $2.3 \%$ of patients [18]

The strengths of our study include a robust unselected prospective design with nationwide harmonized monitoring practices across all participating centers. Although evidence from real-world centers supports the efficacy and safety of ustekinumab therapy in $\mathrm{CD}$, studies from multicenter cohorts with prospective follow-up, especially in biological exposed patient populations, are still limited in the literature. Limitations to our study are the lack of data on endoscopic activity and fecal calprotectin, and the limited availability of blood samples for serum ustekinumab level assessment.

In conclusion, ustekinumab showed good clinical effectiveness in a real-life multicenter cohort of multiple biological exposed $C D$ patients with high rates of complex disease phenotype. A substantial proportion of the patients achieved corticosteroid-free clinical remission and composite clinical and inflammatory biomarker remission in this one-year prospective follow-up. The rate of dose intensification was high and it was introduced early in treatment, coupled with high rates of drug survival after one-year, reflecting a patient population predominantly receiving second or third line biological therapy.

\section{Conflict of interest}

The authors declare that they have no competing interests.

\section{Acknowledgements}

LG and AI were responsible for protocol development, data collection, drafting and revising the manuscript. LG was responsible for data analysis, and drafting the manuscript. KS, KF, IR, TM, TS, ES, PAG, LB, LL, BL, MJ, AP, AV, PS, AF, ZD, TGT and PM equally contributed to the data collection and manuscript revision. LPL and AI were responsible for research planning and result interpretation, and supervised the manuscript preparation. LPL is acting as guarantor of submission. All authors read and approved the final manuscript including the authorship list.

All authors confirmed that this funding statement is correct, complete, and acceptable to the authors.

\section{Financial support}

National Research, Development and Innovation Office (FK 132834 to PS). This work was supported by the ÚNKP-21-4-II New National Excellence Program of the Ministry of Human Capacities, Hungary. Authors declare no other financial support received for this work.

\section{Supplementary materials}

Supplementary material associated with this article can be found, in the online version, at doi:10.1016/j.dld.2021.07.008.

\section{References}

[1] Mannon PJ, Fuss IJ, Mayer L, et al. Anti-Interleukin-12 antibody for active Crohn's disease. N Engl J Med 2004;351(20):2069-79.

[2] Feagan BG, Sandborn WJ, Gasink C, et al. Ustekinumab as induction and maintenance therapy for Crohn's disease. N Engl J Med. 2016;375(20):1946-60.

[3] Rutgeerts P, Gasink C, Chan D, et al. Efficacy of ustekinumab for inducing endoscopic healing in patients with Crohn's disease. Gastroenterology 2018; 155(4):1045-58

[4] Sandborn WJ, Gasink C, Gao LL, et al. Ustekinumab induction and maintenance therapy in refractory Crohn's disease. N Engl J Med 2012;367(16):1519-28.

[5] Satsangi J, Silverberg MS, Vermeire S, et al. The Montreal classification of inflammatory bowel disease: controversies, consensus, and implications. Gut 2006;55:749-53.

[6] Best WR, Becktel JM, Singleton JW. Rederived values of the eight coefficients of the Crohn's disease activity index [\#x005D;. Gastroenterology 1979;77:843-6.

[7] Vermeire S, Schreiber S, Sandborn WJ, et al. Correlation between the Crohn's disease activity and Harvey-Bradshaw indices in assessing Crohn's disease severity. Clin Gastroenterol Hepatol 2010;8:357-63.

[8] Biemans VBC, van der Meulen-de Jong AE, van der Woude CJ, et al. Ustekinumab for Crohn's disease: results of the ICC registry, a nationwide prospective observational cohort study. J Crohns Colitis 2020;14(1):33-45.

[9] Straatmijer T, Biemans VBC, Hoentjen F, et al. Ustekinumab for Crohn's disease: two-year results of the Initiative on Crohn and Colitis (ICC) registry, a nationwide prospective observational cohort study. J Crohns Colitis 2021:jjab081 Epub ahead of print.

[10] Battat R, Kopylov U, Bessissow T, et al. Association between ustekinumab trough concentrations and clinical, biomarker, and endoscopic outcomes in patients with Crohn's disease. Clin Gastroenterol Hepatol 2017;15:1427-34 e2. 
[11] Bar-Gil Shitrit A, Ben-Ya'acov A, Siterman M. Safety and effectiveness of ustekinumab for induction of remission in patients with Crohn's disease: a multicenter Israeli study. United Eur Gastroenterol J 2020;8(4):418-24 Erratum in: United European Gastroenterol J. 2020;8(4):498.

[12] Viola A, Muscianisi M, Macaluso FS, et al. Sicilian Network for Inflammatory Bowel Disease (SN-IBD)". Ustekinumab in Crohn's disease: real-world outcomes from the Sicilian network for inflammatory bowel diseases. JGH Open 2021;5(3):364-70.

[13] Liefferinckx C, Verstockt B, Gils A, et al. Long-term clinical effectiveness of ustekinumab in patients with Crohn's disease who failed biologic therapies: a national cohort study. J Crohns Colitis 2019;13(11):1401-9.

[14] f Björkesten CG, Ilus $T$, Hallinen $T$, et al. Objectively assessed disease activity and drug persistence during ustekinumab treatment in a nationwide real-world Crohn's disease cohort. Eur J Gastroenterol Hepatol 2020;32(12):1507-13.
[15] Iborra M, Beltrán B, Fernández-Clotet A, et al. Real-world long-term effectiveness of ustekinumab in Crohn's disease: results from the ENEIDA registry. Aliment Pharmacol Ther 2020;52(6):1017-30.

[16] Engel T, Yung DE, Ma C, et al. Effectiveness and safety of Ustekinumab for Crohn's disease; systematic review and pooled analysis of real-world evidence. Dig Liver Dis. 2019;51(9):1232-40.

[17] Khorrami S, Ginard D, Marín-Jiménez I. Ustekinumab for the treatment of refractory Crohn's disease: the spanish experience in a large multicentre open-label cohort. Inflamm Bowel Dis 2016;22(7):1662-9.

[18] Adedokun OJ, Xu Z, Gasink C, et al. Pharmacokinetics and exposure response relationships of Ustekinumab in patients with Crohn's disease. Gastroenterology 2018;154(6):1660-71. 